\title{
Pathogenesis and Control of Mangrove Leaf Spot Disease
}

\author{
Bei Liu', Yuming Zhou' ${ }^{2}$ Jian Xue ${ }^{3}$ \\ ${ }^{1}$ Changchun Sci-Tech University, Changchun, Jili, 130600 \\ ${ }^{2}$ Anhua Agricultural Insurance Company Ltd., Changchun, Jili, 130000 \\ ${ }^{3}$ Jilin Agricultural Sciences, Jilin, Jili, 132000
}

Keywords: Leaf Spot Disease, Mangrove Grow, Pathogenesis and Control Method

\begin{abstract}
Mangrove economic value is high, but the mangrove is easily damaged by the leaf spot after the seedlings are damaged, the seedlings are withered, affecting the number and quality of the nursery stock; after the forest trees are damaged, causing the leaves to fall fruit, the spoon shook the tree grow and strong. Mangrove leaf spot disease is mainly harmful to mangrove leaves and shoots, seedlings and young trees are susceptible to disease.
\end{abstract}

\section{Introduction}

Mangrove, arbor or shrubs, 2-4 m tall; bark dark brown. Leaves elliptic to oblong-elliptic, long 7-12 (-16) cm, broadly 3-6 cm, apically apex or apex, base broadly cuneate, midvein red, lateral veins slightly obtuse; Petiole stout, pale red, 1.5-2.5 cm long; stipules long 5-7 cm. Many people see mangroves, a green forest vibrant. Experts explain: in the tropical and subtropical regions of the world, some of the growing plants on the land, into the ocean edge, after a very long evolution process, formed in the intertidal zone of mangroves, which in the tide, The woody plant communities that are periodically submerged by seawater are rich in them. Tannic acid, Was cut down after oxidation into red, so called. The Mangrove has 14 genera, more than 100 species, distributed in Southeast Asia, Africa and the Americas tropical region. Often with Sonneratiaceae (Sonneratiaceae), verbena plants mangrove forest.

\section{Leaf Spot Symptoms and Pathogens}

In this study, Gompertz, Logistic and Exponential FH epidemiological models were used to fit the data obtained from the mangrove field in 2015 by SAS software. The disease index was the dependent variable and the surveyed days were independent variables. The results show that the $F$ value, RMSEF (root mean square error) value of each model, the square R2 value of the correlation coefficient and the probability of probability are compared, and the best fit model is selected (Fu Junfan et al., 2013). As can be seen from the data in Table 3-7, the Gompertz and Logistic models have a good effect on the epidemic dynamics of mangrove leaf spot. Compared with the Logistic model, the Gompertz model has an asymmetric S-curve, and the disease develops slowly and slowly, and the peak is in the front (S, 1986). Comparing the F value, R2 value and RM value of the two models, the Gomp state tz model is superior to the Logistic model, which can reflect the epidemic law of mangrove leaf spot.

The Gompertz model is $\mathrm{Y}=\mathrm{KxEXP}$ for $\mathrm{xEXP}(-\mathrm{rxt})$ ), where $\mathrm{Y}$ is the disease index of mangrove leaf spot at time t; K, B and $r$ are constants. According to Zhu Qiairen's (2002) derivation of the Gompertz model, we can see that the maximum growth rate of the curve is $\mathrm{Y}=\mathrm{Kxr} / \mathrm{e}$ and the inflection point coordinate is $\mathrm{Y}=\mathrm{K} / \mathrm{e}$. The constant value of the formula is into the maximum growth rate of 76, the inflection point disease index of 23.4, 38.1. Comprehensive data on the $\mathrm{W}$ available, the inflection point of the disease index increased on July 31, the disease index growth rate of 0.76 . 
The Gompertz model formula can be deformed as $\mathrm{t}=\ln [(\ln \mathrm{Y}-\ln \mathrm{K}) / \mathrm{-}$ - B] $/$ - r. 5\% and 95\% of the maximum disease index were substituted into the above formula, respectively, to calculate the disease index reached $5 \%$ and $\% \%$ of the time, which derived the mangrove leaf spot epidemic period (Fu Jun Fan et al., 2013). When the disease index reaches 5\%, that is, $\mathrm{Y}=2.8$, can be obtained $\mathrm{t}$ is 2.8; when the disease index to $\% \%$ is, that is, $\mathrm{Y}=53.1$, can be obtained $\mathrm{t} 90.9$. It can be seen that the origin of mangrove leaf spot is June 25, June 25 to September 21 for the Sheng period, September 21 after the recession.

According to the observation of the incidence of disease in the field, the disease situation is generally increased after the rain, which speculated that disease and temperature and humidity are closely related. According to the data recorded by the temperature and humidity recorder, the daily maximum temperature, minimum temperature, average temperature, maximum humidity, minimum humidity, average humidity, accumulation temperature and accumulated humidity were calculated, and the average humidity and humidity values were calculated every 7 days.

The highest temperature, mean temperature and maximum humidity were negatively correlated with the increase of disease index. The lowest temperature, minimum humidity and average humidity were positively correlated with the disease index. The correlation coefficient of each environmental factor is less than $0.4 \%$, while the significant coefficient is greater than 05 , we can see that these six environmental factors are not mangrove leaf spot disease and epidemic dominant factors

According to the follow-up survey of Fushun mangrove cultivation in 2014, the disease initially damaged the underlying leaves, and later spread to the top leaves, leading to premature senescence, early fall off and other serious consequences. The rate of mangrove leaf spot disease was about 30\% in that year, but increased rapidly to $82 \%$ in 2014 , while the disease rate reached $78 \%$. Late onset, the average disease index reached 43.6. Through the continuous investigation of the occurrence of mangrove leaf spot in the pharmacy, the author found that mangrove leaf spot disease occurred more serious after the rain. The reason was mainly due to the large rainfall in late July, the higher the humidity in the field and the higher temperature, And is conducive to the occurrence of disease. In addition, the land for three consecutive years of planting the same product of God, to a certain extent, increased the occurrence and prevalence of Atractylodes leaf spot. The investigation found that the top of the red leaves is not easy to disease, but the underlying leaves of the disease is more serious, the mechanism of its resistance to be further research.

In this study, the prevalence of mangrove leaf spot disease in the medicinal botanical garden was investigated. The disease index increased rapidly in August, reaching a maximum of 55.9, and the maximum rate of disease index was 76. In late September, In this study, three different models (Gompertz, Logistic, S-curve) were fitted to the disease index and the survey period using SAS software. The results showed that compared with the other two models, the Gompertz model could reflect the dynamic situation of the disease index of mangrove leaf spot and increase the field dynamics of mangrove leaf spot. In order to explore the relationship between temperature and humidity and field index, the correlation between accumulation temperature and accumulated humidity and disease index was analyzed by SPSS software. The results showed that the accumulation temperature and accumulated humidity were related to the disease The expansion was significantly correlated. In this study, the single-year incidence of mangrove leaf spot disease was systematically investigated, and the epidemiological model study and the correlation analysis of temperature and humidity were carried out, and the occurrence rule of mangrove leaf spot was clear, the prevention and control of leaf disease provides a theoretical basis.

\section{Study on the Control of Leaf Spot}

In order to expand the scale of Chinese herbal medicine production, increase the economic benefits of Chinese herbal medicine, Chinese herbal medicine production had to pursue high-yielding varieties of large-scale cultivation and its supporting high fertilizer! High pesticide input high yield measures. Needless to say, this high input and high output of the production model, in order to meet the growing demand for Chinese herbal medicines, made a great contribution. 
However, long-term large-scale cultivation of a single variety and the application of pesticides and chemical fertilizers, resulting in the traditional Chinese herbal medicine planting area of the ecosystem is becoming increasingly fragile and serious deterioration of the ecological environment, frequent crop diseases, pathogen differentiation and accelerated growth, disease epidemic The cycle is getting shorter and shorter, Chinese herbal medicine is seriously reduced. This kind of fertilizer and pesticide high input mode of production not only can not effectively control the epidemic of Chinese herbal medicine pests and diseases, but cause the vicious circle of ecosystem, seriously restricts the national production and sustainable development of Chinese herbal medicine. As we all know, ecology is the study of biological and environmental changes in the relationship between science, biological population and its survival environment constitutes an ecological system. Changes in the environment affect the number and balance of biological populations. The number of biological populations and their survival relationship affect the stability of ecosystems. The more the number of biological species is the more stable the ecosystem. On the contrary, the less the number of biological species is the more fragile the ecosystem. Biodiversity-rich areas are generally less prone to catastrophic pests and diseases. The reason is that the ecosystems at all levels of nutrition in the food network are mutually restricted, and any species can not grow indefinitely, so that it is in equilibrium (especially the use of biodiversity to control plant diseases and insect pests, It is the means to return to the natural control of plant diseases and insect pests, with the operation of the original nature and understanding of the advanced nature of it not only can restore the ecosystem into a complex biodiversity of natural ecosystems, but also effective control of crop diseases and pests, Planting and sustainable development strategies.

As we all know, ecology is the study of biological and environmental changes in the relationship between science, biological population and its survival environment constitutes an ecological system. Changes in the environment affect the number and balance of biological populations. The number of biological populations and their survival relationship affect the stability of ecosystems. The more the number of biological species is the more stable the ecosystem. On the contrary, the less the number of biological species have the more fragile the ecosystem. Biodiversity-rich areas are generally less prone to catastrophic pests and diseases. The reason is that the ecosystems at all levels of the food network are constraining each other, and any species can not grow indefinitely, so that it is in equilibrium (Yuming et al., 1999). The use of biodiversity to control plant diseases and insect pests, fundamentally return to the natural control of plant diseases and insect pests means, with the operation of the original and understanding of the advanced nature. It will not only restore the ecosystem into a complex natural ecosystem of biodiversity, but also effectively control crop pests and diseases, and promote the green Chinese herbal medicine planting and sustainable development strategy.

At present, there are few varieties of immunity, but the disease resistance between varieties is obvious. Features are raw materials. Inbred lines are not resistant to disease, the breeding of hybrids are not resistant to disease, so the key to cultivating disease-resistant varieties is to identify and cultivate resistant inbred lines. Planting disease-resistant varieties is a big control. Effective measures of leaf spot. From the North China point of view, the current production of the promotion of varieties of disease resistance difference is more obvious. Selection of disease-resistant varieties according to local conditions, should also pay special attention to local areas to more varieties with a reasonable mix.

Elimination of overwintering bacteria source, crop rotation can reduce the amount of bacteria. Corn harvest is after the complete removal of field disease debris, and timely deep turn, which is an important measure to reduce the source of bacteria. Crop rotation. Clean pastoral, conditional can be more than two years of rotation. After harvesting in time to plow, stored corn straw should be disposed of in advance before sowing to reduce the initial source of infection.

\section{Conclusion}

Based on the above results, it was found that $50 \%$ of the bacteriophage powder and $300 \mathrm{~g} /$ According to the relevant study, some fungi can start the bypass oxidation pathway to complete the 
electron transfer, thus showing the resistance to the drug. However, due to the presence of free oxygen scavengers in the plant, the activation of bypass oxidation can be prevented. Therefore, although the different concentration of $300 \mathrm{~g} / \mathrm{L}$ Velvet vinegar pear agent in the culture medium on the low inhibitory rate, but the field control effect is better. It is combined with the author of the results of field investigation of the disease of the herbaceous leaf spot, the prevalence of disease infection and the initial screening of the drug, that the prevention and treatment of the disease should also be the main prevention. As the focus of work, a comprehensive disease prevention and control work. In order to effectively prevent the epidemic of stem spot leaf spot, we should strengthen the field management, timely removal of field disease residue, increase the micro-fertilizer to improve plant resistance, strengthen the disease monitoring, in the early onset of timely application, Prevention and control.

\section{Acknowledgements}

Correlative Study on the Identification, Pathogenesis and Drug Sensitivity of Phyllostachys heterocycla var Proj. No. 20160426

\section{References}

[1] Hu Jiacheng, Zhao Jingyuan. Progress in detection technology of Phytophthora infestans [J]. Chinese Journal of Plant Pharmacy, 2014 (03)

[2] He Jingjing, Zhou Rujun. Study on indoor virulence and field control of different fungicides on peanut net spot bacteria [J]. Chinese Journal of Oil Crop Sciences, (2015)

[3] Ma Cheng, Peng Lijuan, Wu Shiping. Identification of Pathogens of Leaves of Leaves of Ginkgo Leaves [J]. Guizhou Agricultural Sciences, 2014 (12)

[4] Wang Guangda, Huang Chunan. Study on the Relationship between Resistance of Different Maize Varieties to Leaf Spot Disease and Related Defensive Enzyme Activities [J]. Maize Science, 2014 (05)

[5] Fu Junfan. Identification of pathogen and its biological characteristics of anthracnose in Shandong yam [J]. Journal of Jilin Agricultural University, 2014 (04)

[6] Zhou Rujun, Xu Haijiao, Fu Junfan. Identification of pathogen of Sclerotia sclerotiorum and its biological characteristics [J]. Journal of Shenyang Agricultural University, 2014 (03) 